\title{
The interaction of migration range, regional economic development level on social capital and public health services of internal migrants: evidence from the China Migrant Dynamic Survey in 2017
}

\author{
Zhen Yang \\ Tongji University \\ Cheng-hua Jiang ( $\sim$ jchtongji@163.com ) \\ Tongji University \\ Jiansheng Hu \\ Jinggangshan University
}

\section{Research Article}

Keywords: Internal Migrants, Migration Range, Regional Economic Development Level, Social Capital, Public Health Services

Posted Date: November 12th, 2021

DOl: https://doi.org/10.21203/rs.3.rs-309989/v5

License: (c) (1) This work is licensed under a Creative Commons Attribution 4.0 International License.

Read Full License 
Title page

The interaction of migration range, regional economic development

level on social capital and public health services of internal migrants: evidence from the China Migrant Dynamic Survey in 2017

Zhen Yang ${ }^{1,2}$, Cheng-hua Jiang ${ }^{1, *}$, Jiansheng $\mathrm{Hu}^{2}$

${ }^{1}$ School of medicine, Tongji university, Shanghai 200092, China;

${ }^{2}$ School of medicine, Jinggangshan university, Jian,Jiangxi 343009, China

*correspondence:Cheng-hua Jiang

School of medicine,

Tongji university,

No 1239 Siping Road, Yangpu District,

Shanghai 200092,

China

Phone: +8618917266778

E-mail:jchtongji@163.com 


\title{
The interaction of migration range, regional economic development \\ level on social capital and public health services of internal migrants: evidence from the China Migrant Dynamic Survey in 2017
}

\begin{abstract}
Background Studies have confirmed that migration range (MR), regional economic development level (REDL) and social capital have impacts on the internal migrants' (IMs') accessibility of National Essential Public Health Services (NEPHS), but no research has explored the interaction of the four variables.
\end{abstract}

Method A cross-sectional sample of 115412 IMs from the China Migrant Dynamic Survey in 2017 was selected. The sampling method was layered, multi-stage, and probability proportional to size. Logistic regression was conducted by SPSS22.0, sex, residence duration, community type, and education as the control variables, REDL and MR as the moderating variables, social capital as independent variable and awareness of NEPHS and registration of health records (RHR) as the dependent variables.

Results In high income provinces (HIPS), the intra-provincial IMs' CSC, civic participation, social participation, NEPHS awareness and RHR were $4.73 \pm 1.736,49.3 \%, 55.3 \%, 61.1 \%$, and $31.9 \%$, and the inter-provincial IMs' levels were 4.01士1.713, 40.5\%, 47.2\%, 54.6\%, and 26.8\%; In low-and middle- income provinces (LMIPs), the intra-provincial and inter-provincial IMs' levels were $5.13 \pm 1.767,46.1 \%, 48.1 \%, 65.6 \%, 35.5 \%$, and $5.02 \pm 1.775,41.5 \%, 41.5 \%, 62.06 \%, 34.1 \%$, respectively. In the complete regression model, the interaction of MR, REDL and CSC was not significant, while the interaction of MR, REDL and SSC was significant. In the group regression model, the ORs of CSC, civic participation, and social participation were significantly greater than 1 both in HIPs and LMIPs. However, the interaction of MR and SSC was significant in HIPs but not significant in LMIPs.

Conclusions The social capital and NEPHS utilization of inter-provincial IMs was significantly lower than that of intra-provincial IMs, and these gaps were more prominent in HIPs. Social capital had a positive effect on IMs' NEPHS utilization, and this effect was significantly moderated by MR and REDL. The next focus of the equalization of public services should be the primary health resources supply and the IMs' social capital construction of HIPs.

Keywords: Internal Migrants, Migration Range, Regional Economic Development Level, Social Capital, Public Health Services

\section{Background}

Social development drives global mobility. Migration can be internal or international, and the internal migrants (IMs) are about four times the size of international migrants [1]. Due to institutional restrictions, IMs in some low - and middle- income countries (LMICs) are not treated equally as natives, and they need to face many obstacles in health resources utilization. Studies from China, India, Indonesia and other LMICs show that the IMs have insufficient access to local public health services [2-6], and this has become a risk affecting the health of IMs [2]. How to 
effectively improve the IMs' accessibility to health services has become an important challenge for LMICs. One way to address this challenge is to explore the influencing factors and how they work.

Social capital is a social determinant of health [7], it can influence individual health outcomes by influencing access to health services [8]. Different types of social capital affect health services utilization by influencing the availability of health services in communities, the availability and effectiveness of outreach resources between health-care providers and communities they serve, and care-seeking behavior of individuals in those communities [9]. Migration means a loss of the original social network and a reduction of social participation in the new environment [10]. Surveys show that migrants generally lack social capital, which limits their access to local health services[11, 12]. Perkins et al [13] pointed out that social capital may be particularly vital to health outcomes as extended webs of social ties often are the principal source of various resources in LMICs. Therefore, social capital may play a prominent role in IMs' access to health services in LMICs, and some relevant studies support this point [6, 14-18 ].

Not everyone has access to the same sources of social capital and not everyone will benefit in the same way [19]. Discussion of the relationship between social capital and health must be set in context to be more effective. Carpiano and Moore [20] suggested that social capital and health knowledge base can be better served by asking three foundational questions of (a) how, (b) for whom, and (c) in which contexts does social capital work, rather than focusing primarily on whether social capital provides some universal health benefit. In fact, existing studies on the relationship between social capital and IMs' accessibility to health services have not considered these three questions comprehensively. The relationship between social capital and health is not consistent across countries [21], which could be attributed to the differences in economy, culture and system among these countries [22]. Similarly, there are differences in regional economic development levels (REDL) and cultural practices in different regions of the same country. These gaps are likely to influence the relationship between social capital and access to health services, but few studies have demonstrated this.

During the past three decades, China has experienced the largest migration in human history, with hundreds of millions of rural inhabitants moving temporarily or permanently to cities. Internal migration is inevitable and essential for the economic and social prosperity of China. IMs exceeded 240 million in 2017 [23], and the number was stable with a slight decline in recent years. In 2009, the central government initiated the National Essential Public Health Services (NEPHS), the project is provided free of charge to all residents, including IMs who have lived there for more than six months [24]. NEPHS includes health records, health education, immunization for children, and chronic disease management etc. Since 2009, the government has successively introduced measures to strengthen the equalization of NEPHS [25-27]. Efforts have paid off, and the IMs' NEPHS utilization level is rapidly improving [28]. The new situation promotes the government's working mode to change from flooding to precise [27], and the focus has gradually shifted to how to achieve the NEPHS equalization within the IMs. Researchers are beginning to conduct more studies of population differentiation[3, 4, 14, 15, 28-33].

Existing studies have confirmed that both migration range (MR) and REDL have a significant impact on NEPHS utilization. Studies have consistently concluded that the NEPHS utilization of inter-provincial IMs is lower than that of intra-provincial IMs [3, 28-33]. However, opinions on the relationship between REDL and IMs' NEPHS utilization are inconsistent: some believe that the 
IMs' NEPHS utilization in high income provinces (HIPs) is lower than that in low- and middle-income provinces (LMIPs) [28-33], but Yang Xin [4] does not think so. Surprisingly, these studies almost analyzed MR and REDL as independent factors, without considering possible interactions between the two variables. On the other hand, studies provide important clues for further exploring the relationship among MR, REDL and social capital. International migrants research suggests acculturation affects the social network construction of migrants [34]. Among factors that affect the social integration of migrants, cultural exclusion is an important source of prejudice, discrimination and social isolation suffered by individuals in the process of migration [35]. In fact, dialects, diets and customs are different among provinces in China, and the problem of acculturation of IMs also exists. However, there are few studies on the relationship between acculturation and social capital of IMs. Yang and Zhang [36] found that being familiar with dialect can help IMs better integrate into society, while Xiao et al. [37] found that the IMs in HIPs has the lowest level of psychological integration. However, studies do not provide direct evidence that MR and REDL affect social capital.

To sum up, given MR and REDL may have a significant impact on the IMs' social capital and health services utilization, and social capital is closely related to health services utilization, we speculate that different MR and REDL create different contexts for IMs, and these different contexts may significantly affect the mechanism of social capital on service utilization. To test this hypothesis, we adopted a retrospective cross-sectional survey, a sample of the China Migrant Dynamic Survey in 2017 was analyzed, and we want to verify four hypotheses: (1) IMs' social capital may be significantly affected by MR and REDL; (2) MR and REDL have a significant interaction on IMs' NEPHS utilization; (3) Social capital has a significant impact on the IMs' NEPHS utilization; (4) The relationship between social capital and NEPHS utilization of IMs is significantly moderated by MR and REDL. This study was the first to explore the impact of MR and REDL on IMs' access to public health services from the perspective of social capital based on a national sample. Our study can provide evidence for the Chinese government to deepen the equalization of NEPHS for IMs, and can also provide references for other LMICs to deal with the health problems of migrants.

\section{Methods}

\section{Data}

A retrospective cross-sectional study was used in this study. The data was obtained from the China Migrant Dynamic Survey in 2017 provided by the Migrant Population Service Center. China Migrant Dynamic Survey is an annual national sample survey of the IMs organized by the National Health Commission from 2009, with an annual sample size of approximately 200000 households. China Migrant Dynamic Survey adopts the layered, multi-stage, and probability proportional to size (PPS) sampling method. This study adopted the individual questionnaire A of China Migrant Dynamic Survey, which was uniformly printed and distributed by the National Health Commission. The questionnaire A includes basic information about respondent's demography, perception of the destination, the state of social interaction, and utilization status of NEPHS, etc. Full-time investigators collected the questionnaire data through household interviews, and each respondent gave informed consent before commencing the interview. Dates were entered through the migrant population health and household planning dynamic monitoring system, input data was subjected to multiple checks to ensure quality. 
The target population of China Migrant Dynamic Survey is the inflow population aged 15 and above who came to live in the local area (county or city) one month before the survey. In this study, the inclusion conditions of sample were set as "18-59 years of age, residence duration more than one year". Beijing, Tianjin and Shanghai, the three cities have only inter-provincial IMs, which do not meet the objective of this study, so the samples of these three cities were excluded. After the quality audit, 115412 people were finally included. In addition, we introduced GDP per capita to reflect the REDL of each provincial region, and GDP per capita is based on 2017 data from the National Bureau of Statistics.

\section{Measurement}

\section{Utilization of NEPHS}

Awareness of NEPHS is a prerequisite for NEPHS utilization [3]. Awareness of NEPHS was set as an outcome variable, and the question was "Have you heard of the NEPHS?" and the answer was "yes or no". Another outcome variable was registration of health records (RHR). RHR is one of the service priorities and reflects the actual utilization of NEPHS by the IMs. IMs can voluntarily register for a health record account at the local community health service center, and their health information will be recorded. The question was "Have you registered health records at the destination?" and the answer was "yes or no".

\section{Soical Capital}

Social capital refers to the resources and benefits received through connections with others, either as individuals or groups, it can be distinguished into two dimensions: cognitive social capital (CSC) and structural social capital (SSC) [7]. The CSC generally refers to IMs' perceptions, beliefs, and attitudes toward their destination, with corresponding measures focused mainly on the concepts of generalized and particularized trust [38]. In this study, social capital was limited to the destination, and it was a localized social capital that reflects the social resources available to the IMs there. There were six questions in the survey: "I like the city/place I live now", "I am concerned about the changes in the city/place I live now", "I am very willing to blend with the local people and become a part of them", "I think the local people are willing to accept me as a part of them", "I feel locals look down on outsiders" (reverse scoring) and "I feel like I'm already a local". The answer to each question was " $1=$ totally disagree, $2=$ disagree, $3=$ basically agree, and $4=$ totally agree". The internal consistency coefficient $\alpha=0.786$. According to the distribution of scores, CSC was divided into eight levels: 1 (6-14 points), 2 (15-16 points), 3 (17 points), 4 (18 points), 5 (19-20 points), 6 (21-22 points), 7 (23 points), and 8 (24 points).

SSC refers to the presence of formal opportunity structures or activities in which individuals build or strengthen their social connections [38]. The SSC of this survey included civic participation and social participation in the destination. Questions of the former were: Since 2016, "have you made suggestions to your unit/community/village or supervised the unit/community/ village affairs management?", " have you participated in property donation, blood donation, volunteer activities, etc.?", "have you reported the situation/put forward policy suggestions to relevant government departments in various ways?", "have you posted online comments on national affairs and social events or participated in related discussions?", "have you participated in party/youth league organization activities and party branch meetings?". Respondents were assigned a "yes" if they participated in any of these tasks, and a "no" if they did not. The question of social participation was "Have you participated in any of the following activities in the past year: trade unions, volunteer associations, homecoming associations, fellow-students association, 
home town chamber of commerce, others?". Respondents were assigned a "yes" if they participated in any of these organizations, and a "no" if they did not.

Demographic variables

As mentioned above, MR and REDL variables have significant impacts on social capital and service utilization. Meanwhile, studies have also confirmed that sex, education, community type, residence duration, have significant impacts on IMs' NEPHS [3, 4, 28-33]. So all of these variables are included in the analysis. Education was divided into two categories: $\leqslant 9$ and $>9$ years groups. The residence duration was divided into three groups: $\leqslant 3$ years, 3-10 years and $\geqslant$ 10 years. Community types were divided into urban and rural communities. MR was divided into inter-provincial and intra-provincial migration. REDL was divided into two groups according to per capita GDP in 2017, five provinces with a per capita GDP of more than RMB 70,000 were classified as HIPs, and the other 23 provinces were classified as LMIPs.

\section{Statistical analysis}

First, we described the distribution characteristics of all the included variables (table 1). Secondly, cross-table and chi-square tests were used to verify the influence of sex, education, residence duration, community type, MR and REDL on IMs' awareness of NEPHS and RHR (table 2). Thirdly, we examined the interaction of MR and REDL on the IMs' social capital and NEPHS utilization, statistical methods including two-way ANOVA and logistic regression analysis (table 3, 4). Fourthly, we used sex, residence duration, education, community type as the control variables, MR and REDL as the moderating variables, and awareness of NEPHS and RHR as the dependent variables for a hierarchical logistic regression analysis (table 5) to discuss the degree and direction of the interaction of MR, REDL, social capital on IMs' NEPHS utilization. Finally, we conducted grouping logistic regression according to REDL (table 6), sex, residence duration, community type, and education as the control variables, MR as the moderating variables, and awareness of NEPHS and RHR as the dependent variables. Sampling weights were included in all analyses to adjust for the complex survey design. In logistic regression analysis model, Odds ratios (OR) were presented. All the analyses were performed using SPSS 22.0.

\section{Results \\ Characteristics of the sample}

Table 1 shows that the NEPHS utilization level of IMs is low. The awareness of NEPHS was $59.0 \%$, while the RHR rate was even lower to $30.1 \%$. In terms of sample composition, the proportion of migrant population was higher among males, urban communities, residents duration $>3$ years, years of education $\leq 9$, inter-provincial migrants and those from HIPs. The IMs' CSC was high, $79.9 \%$ of IMs had a positive evaluation of the destination ( $\geq 1$ ppoints), but IMs' SSC was low. In the past year, 56.4\% of them did not participate in the listed civic activities, and $51.5 \%$ did not participate in the listed organizational activities.

Table 1 Characteristics of the sample, in 2017, China ( $N=115412)$.

\section{Results of univariate analysis}

Table 2 shows that sex, community type, residence duration, education, MR, REDL and social capital all have significant impacts on IMs' NEPHS utilization. The awareness of NEPHS and RHR of inter-provincial IMs were significantly lower than those of intra-provincial IMs. 
Compared with LMIPs, the IMs' rates of NEPHS awareness and RHR in HIPs were significantly lower. The impact of social capital, civic participation and social participation on NEPHS utilization was more prominent than other variables. With the increase of CSC, the NEPHS utilization level of IMs also increased, but the relationship is nonlinear.

Table 2 Impact of sex, community type, community type, education, MR, REDL and social capital on utilization of NEPHS (N=115412).

\section{Interaction of MR and REDL on social capital and utilization of NEPHS}

According to table 3, we found that there was a significant difference in the IMs' social capital between different REDL groups. The IMs of HIPs had lower CSC $(t=77.268, p<0.001)$ and civic participation $\left(X^{2}=22.258, p<0.001\right)$ than those of the IMs of LMIPs, but the former had higher social participation $\left(\mathrm{X}^{2}=128.583, \mathrm{p}<0.001\right)$. The IMs' NEPHS utilization level was also significantly different between HIPs and LMIPs, the NEPHS awareness $\left(X^{2}=627.718, p<0.001\right)$ and RHR $\left(\mathrm{X}^{2}=388.239, \mathrm{p}<0.001\right)$ of the former were lower than those of the latter. At the same time, we can also find that the level of intra-provincial IMs in the three dimensions of social capital was significantly better than that of inter-provincial IMs, and this gap was greater in HIPs. The interaction among MR, REDL and NEPHS Utilization was similar.

Table 3 Distribution of social capital and NEPHS utilization levels in different MR and REDL groups (N=115412).

Table 3 visually describes the influence of MR and REDL on social capital and NEPHS Utilization, and then we further analyze the direction and intensity of this effect. Two-way ANOVA results showed that the main effect of MR ( $\mathrm{F}=645.074, \mathrm{p}<0.001)$ and REDL $(\mathrm{F}=3187.650$, $\mathrm{p}<0.001$ ) on CSC was significant, and the interaction effect of MR and REDL on CSC was also significant $(\mathrm{F}=658.215, \mathrm{p}<0.001)$. Since civic participation, social participation, awareness of NEPHS and RHR were dichotomous variables, we took these four variables as dependent variables, MR, REDL and MR*REDL as independent variables for logistic regression analysis. Table 4 shows that the main effects of MR on four dependent variables were all significantly negative, the main effects of REDL on four dependent variables were also significant but the direction was not consistent. MR*REDL had significant negative effects on CSC, civic participation, social participation, and awareness of NEPHS, but had no significant effect on RHR.

Table 4 Logistic regression results of MR, REDL and MR*REDL on social capital and utilization of NEPHS $(\mathrm{N}=115412)$.

Table 5 shows that when awareness of NEPHS was taken as the dependent variable, block 2 compared with block 1, Omnibus test $\mathrm{X}^{2}$ increased from $2729.821 \quad(\mathrm{p}<0.001)$ to 8680.254 $(\mathrm{p}<0.001)$, Cox \& Snell $\mathrm{R}^{2}$ increased from 0.023 to 0.072 , Hosmer \& Lemeshow test $\mathrm{X}^{2}$ decreased from $63.315(\mathrm{p}<0.001)$ to $5.954(\mathrm{p}>0.05)$. With RHR as the dependent variable, block 2 compared with block 1, Omnibus test $\mathrm{X}^{2}$ increased from $2195.950(\mathrm{p}<0.001)$ to $6484.332(\mathrm{p}<0.001)$, Cox \& Snell $\mathrm{R}^{2}$ increased from 0.019 to 0.055 , Hosmer \& Lemeshow test $\mathrm{X}^{2}$ decreased from 209.463 $(p<0.001)$ to $23.646(p<0.01)$. Obviously, with the introduction of social capital, the explanatory power and fitting degree of the model both have been significantly improved. When controlling 
for other variables, CSC, civic participation and social participation all had a positive impact on NEPHS utilization. In the regression model with awareness of NEPHS as the dependent variable, THE OR values of MR*CSC, REDL*civic participation, MR*REDL*civic participation and MR*REDL*social participation were significant. In the regression model with RHR as the dependent variable, the OR values of REDL*social participation, MR*REDL*civic participation and MR*REDL*social participation were significant. However, the OR value of MR*REDL*CSC was not significant in both regression models.

Table 5 Logistic regression results of sex, community type, residence duration, MR, REDL and social capital on NEPHS utilization ( $\mathrm{N}=115412)$.

To further clarify the relationship between MR, REDL, social capital and NEPHS utilization presented in table 5. We conducted grouping logistic regression analysis according to REDL (table 6). In model 1 , the omnibus test $\mathrm{X}^{2}$ was 1963.520 ( $\mathrm{p}<0.001$ ), Cox \& Snell $\mathrm{R}^{2}$ was 0.054 , Hosmer $\&$ Lemeshow $\mathrm{X}^{2}$ was $13.431(\mathrm{p}>0.05)$. In Model 2, the corresponding three indexes were 6217.905 $(\mathrm{p}<0.001), 0.075,20.740(\mathrm{p}<0.01)$. In model 3, the corresponding three indexes were 1289.293 $(p<0.001), 0.036,10.105(p>0.05)$. In model 4 , the corresponding three indexes were 5074.320 $(\mathrm{p}<0.001), 0.061,43.297(\mathrm{p}<0.001)$. The value of Cox \& Snell $\mathrm{R}^{2}$ was always larger in the HIPs. Table 6 showed that the situation of Model 1 was similar to model 3, that was, the three main effects of social capital were significantly positive. In Model 2, the three main effects and three interaction effects of social capital were significantly positive, while in Model 4, the situation was very similar, the three main effects and two interaction effects were significantly positive. Combined with the information in table 5 and 6 , MR and REDL had a significant moderating effect on the relationship between SSC and NEPHS utilization.

Table 6 Logistic regression results of sex, community type, residence duration, education, MR and social capital on awareness of NEPHS and RHR in two groups of REDL ( $N=115412)$.

\section{Discussion}

There were three main findings:(1) The social capital level of the inter-provincial IMs was significantly lower than that of the intra-provincial IMs, and the gap was larger in the HIPs. (2) The NEPHS utilization level of inter-provincial IMs was significantly lower than that of intra-provincial IMs, and the gap was larger in HIPs too. (3) The social capital of IMs had a positive impact on their NEPHS utilization, and the relationship between SSC and NEPHS utilization was significantly moderated by MR and REDL.

This study found that the social capital of IMs was characterized by high CSC and low SSC, which was consistent with previous studies [33, 39, 40]. Yip et al. have pointed out that high CSC and low SSC may be a universal characteristic of Chinese people [41]. Migration means a loss of the original social capital for migrants [10], and this loss may be even more pronounced on the inter-provincial IMs. We found that the average level of social capital of inter-provincial IMs was lower than that of intra-provincial IMs. Bourdieu pointed out that culture is one of the key factors affecting social capital [22]. Malacculturation can lead to loss of social networks [34]. China has a vast territory, and there are often visible differences in dialects, diets and customs among provinces. This cultural difference makes it easier for people in the same province to identify with 
each other. A survey [36] finds that, compared with those who have migrated to areas with the same dialect as those of their origin, those who have migrated to areas with different dialects have greater difficulty in social integration. This can also be verified by the lower CSC of the inter-provincial IMs in this study.

REDL also had a significant impact on the social capital of IMs. IMs in HIPs and those in LMIPs face different macro institutional environments. Xiao et al. [37] argued that, compared with other regions, IMs from HIPs have the lowest level of psychological integration, but their level of economic integration and political integration is not low. We have some similar results to this study that the CSC of the IMs in HIPs was significantly lower than that of the IMs in LMIPs, but the difference in SSC between the two groups was not the case. However, it is noteworthy that MR difference of social capital was larger in HIPs, and there is no direct or indirect explanation for this phenomenon in existing studies. According to a survey [23], the HIPs attract more than $54.8 \%$ of the country's migrant population, of which $78.2 \%$ are inter-provincial migrants. In order to control the population size, HIPs have introduced strict household registration access standards. The inter-provincial IMs in HIPs face the dual difficulties of acculturation and institutional exclusion when constructing social capital, which may be one of the reasons why MR gap of IMs' social capital is larger in HIPs.

This study supports the conclusion that the NEPHS utilization level of inter-provincial IMs is significantly lower than that of intra-provincial IMs [3, 28-33]. With regard to REDL, we support that the NEPHS utilization of IMs in HIPs is low [28-33]. Some studies believe that the low level of NEPHS utilization in HIPs is due to the insufficient supply [28, 31]. NEPHS is mainly provided by primary health institutions. Thanks to the support of the central government, primary medical service resources in LMIPs are also abundant, and even have advantages in per capita resources [42]. Our findings provide new evidence for this assertion: the NEPHS utilization of both inter-provincial and intra-provincial IMs was lower in HIPs than in LMIPs. Therefore, we believe that although the higher proportion of inter-provincial IMs (78.2\%) would affect the overall NEPHS utilization level of the HIPs group, the shortage of per capita resources in these regions may be one of the main reasons for limiting the NEPHS level of the IMs. So it is urgent for the HIPs to rapidly increase their primary health resources to address the shortage of NEPHS supply.

The conclusion of this study on the relationship between social capital and NEPHS utilization was consistent with previous research [6, 14-18]. Social capital has an important informational function, and it can influence health information through three mechanisms: increased information exposure, enhanced seeking abilities, and reinforced health culture or norms embedded in social networks [43]. Therefore, social capital had a greater impact on awareness of NEPHS than RHR. We can also see that CSC had a lower impact on NEPHS utilization than SSC, both of which were lower than we expected, and the reasons for this maybe: (a) High CSC of migrant population leads to ceiling effect; (b) The IMs' social network is homogeneous and closed [44], so their SSC quality is poor [45]. The explanatory power of social capital to IMs' NEPHS utilization was smaller in LMIPs and larger in HIPs. Viswanath et al. [46] found a stronger relationship between social capital and access to health information in socioeconomic disadvantaged communities. Perkins et al. [13] noted that individuals in LMICs rely more on social networks to access health services. This study found that social capital played a more important role for inter provincial IMs in HIPs than LMIPs. A more general conclusion might be that the impact of social capital on access to health services is more important in under-resourced areas. 
This study also found that social capital has both a main effect and buffer effect on IMs' NEPHS utilization in HIPs, but only main effect in LMIPs. The latter is in line with Cohen and Wills' points [47], while the former supports Fried and Tiegs' conclusion [48]. The original reason may be that the gap in the social capital of inter-provincial and intra-provincial migrants is small in LMIPs and large in HIPs. MR is related to acculturation, while REDL reflects the intensity of institutional exclusion, so MR and REDL essentially reflect the IMs' socioeconomic status. Uphoff et al [19] proposed three paths by which socioeconomic status could affect the relationship between social capital and health outcomes: (a) A more significant social capital benefit on the health of disadvantaged persons in society, and no effects or limited health benefits for those in positions higher up in the social ladder. (b) People with a low socioeconomic status will generally have less social capital, and the capital available to them cannot be used effectively for health benefits. (c) Social capital might benefit the better-off in society while excluding people with a lower socioeconomic status. Obviously, the relationship between social capital and health services of IMs in HIPs conformed to path a.

\title{
Strength and Limitations
}

From the perspective of ecology, this study comprehensively examined the interaction mechanism among environmental factors (REDL, MR), individual factors (individual social capital) and NEPHS utilization of IMs through a national sample. This study also revealed that the inter-provincial IMs in HIPs are in a disadvantageous position in social capital and NEPHS utilization, which provides a breakthrough for the subsequent equalization of public services for the IMs. Our study provides valuable clues for further exploring the relationship between acculturation, institutional exclusion, resources supply and IMs' health service utilization. Our findings also provide useful implications for other LMICs in dealing with the equalization of health services for IMs. However, this study also had two limitations: First, we used cross-sectional survey data in 2017, which could not reflect the latest status of China's IMs and made it difficult for us to make convincing causal inference about the path of variables. Subsequent studies need to pay attention to the change trend of these variables in the time dimension. Secondly, we only found the possible relationship between MR and acculturation, REDL and institutional exclusion, resource supply, but could not provide direct evidence. Subsequent studies need to improve the measurement of variables.

\section{Conclusions}

The level of social capital and NEPHS utilization of inter-provincial IMs was significantly lower than that of intra-provincial IMs, and these gaps were more prominent in HIPs. Social capital had a significant positive effect on NEPHS utilization of IMs. In LMIPs, SSC had only a main effect, while in HIPs, SSC had both a main effect and buffer effect. We believe that SSC is more important for people of lower socioeconomic status to access health services in resource-poor settings. Therefore, the next step of equalization of public services for IMs should focus on HIPs. One is to increase the supply of grass-roots health services; the other is to pay attention to the construction of social networks for inter-provincial IMs.

\author{
Abbreviations \\ CSC: Cognitive Social Capital
}


RHR: Registration of Health Records

HIPs: high income provinces

IMs: Internal Migrants

LMICs: low- and middle- income Countries

LMIPs: low- and middle- income provinces

MR: Migration Range

NEPHS: National Essential Public Health Services

REDL: Regional Economic Development Level

SSC: Structural Social Capital

\section{Declarations}

\section{Ethics approval and consent to participate}

The "National Internal Migrant Dynamic Monitoring Survey, 2017" data is publicly available to authorized researchers who have been given permission by the Migrant Population Service Center, and written informed consents were obtained from all participants. The analysis of public access data was exempted by the local IRB; as this involved analyzing de-identified existing data, ethical approval was not required.

\section{Consent for publication}

Not applicable.

\section{Availability of data and materials}

Since the data used in this paper were provided by the Migrant Population Service Center, which is the top agency governing migrant population issues in China, we had to sign a legally binding agreement with the agency that we will not share any original data with any third parties. However, interested researchers can apply for access to the data at http://www.ldrk.org.cn/.

\section{Competing interests}

No competing interests in this study.

\section{Funding}

No.

\section{Authors' contributions}

All authors participated in the design of the study. $\mathrm{Z}$ Y carried out the statistical analysis and composed the first draft. $\mathrm{CH} J$ gave opinions for modification. JS $\mathrm{H}$ was responsible for data sorting and manuscript editing. All authors read and approved the final manuscript.

\section{Acknowledgments}

The authors thank the Migrant Population Service Center, National Health Commission of the People's Republic of China for providing the data.

\section{Authors' Information}

Zhen Yang. (1) School of medicine, Tongji university, Shanghai 200092, China;

(2) School of medicine, Jinggangshan university, Jian, Jiangxi 343009, China.

Chenghua Jiang. (1) School of medicine, Tongji university, Shanghai 200092, China.

Jiansheng Hu. (2)School of medicine, Jinggangshan university, Jian, Jiangxi 343009, China.

\section{References}

1. United Nations Development Programme. Human Development Report 2009: Overcoming barriers: Human mobility and development. 
2. Gong P, Liang S, Carlton EJ, et al. Urbanization and health in China. The Lancet. 2012; 379(9818): 843 - 852.

3. Guo J, Shao F, Fan $\mathrm{H}$, et al. Analysis on the access to the basic public health care services and influencing factors among migrants.Chinese Journal of Health Policy. 2016; 9(8): 75-82.

4. Yang $X$. Difference in utilization of basic public health service between registered and migrant population and its related factors in China,2015. Chin J Public Health. 2018; 34(10):781-785. doi:10.11847/zgggws1115819.

5. Kusuma KS, Babu BV. Migration and health: A systematic review on health and health care of internal migrants in India. Int J Health Plann Mgmt. 2018; 33:775 - 793.

6. Lu Y. Rural-urban migration and health: Evidence from longitudinal data in Indonesia. Social Science \& Medicine. 2010; 70 (2010): $412-419$.

7. Kawachi I, Subramanian SV, Kim D. Social capital and health. New York: Springer social capitalience + Business Media, LLC; 2008.

8. Drose KP, Varda DM. Social capital and health care access: A systematic review. Med Care Res Rev. 2009; 66(3): 272-306. doi:10.1177/1077558708330428.

9. Derose, KP. social capital: What's it good for? Exploring the relationship between community social structure and access to health care. Los Angeles: University of California. 2003. (Dissertation)

10. Palloni A, Massey DS, Ceballos M, et al. Social capital and international migration: A test using information on family networks. American Journal of Sociology. 2001; 106(5): 1262 - 1298.

11. Deri C. Social networks and health service utilization. J Health Econ. 2005; 24: 1076 - 107.

12. Devillanova C. Social networks, information and health care utilization: Evidence from undocumented immigrants in Milan. Journal of Health Economics. 2008; 27 (2008): 265 - 286.

13. Perkins JM, Subramanian SV, Christakis NA. Social Networks and Health: A systematic review of sociocentric network studies in low- and middle-income countries. Soc Sci Med. 2015; 125: 60-78. doi:10.1016/j.socsci med.2014.08.019.

14. Hou ZY, Lin S, Zhang D. social capital, neighbourhood characteristics and utilization of destination public health services among domestic migrants in China: a cross sectional study. BMJ OPEN. 2017; 7: e014224. doi:10.1136/bmjopen-2016-014224.

15. Zeng CB, Li XM, Du HF, et al. Experiences of stigma and health service utilization among young rural-to-urban migrants in China: the mediation role of Social capital. Vulnerable children and youth studies. 2020; 15(2): 97-109.

16. Kusuma YS, Kumari R, Kaushal S. Migration and access to maternal healthcare: Determinants of adequate antenatal care and institutional delivery among socio-economically disadvantaged migrants in Delhi, India. Trop Med Int Health. 2013; 18(10): 1202-1210.

17. Gawde NC, Sivakami M, Babu BV. Utilization of maternal health services among internal migrants in Mumbai, India. J Biosoc Sci. 2016; 48(06): 767-796.

18. Santalahti M, Sumit K, Mikko Perkiö. Barriers to accessing health care services: a qualitative study of migrant construction workers in a southwestern Indian city. BMC Health Services Research. 2020; 20: 619.

19. Uphoff EP, Pickett KE, Cabiese B, et al. A systematic review of the relationships between Social capital and socioeconomic inequalities in health: a contribution to understanding the psychosocial pathway of health inequalities. International Journal for Equity in Health. 2013; 12(1):54.

20. Carpiano RM, Moore S. So What's Next? Closing thoughts for this special issue and future steps for social capital and public health. Soc Sci Med. 2020; doi.org/10.1016/j.socsocial capitalimed.2020.113013.

21. Jiang N, Wu B, Lu N, et al. Neighborhood-based social capital and cognitive function among older adults in five low- and middle-income countries: Evidence from the World Health Organization Study on global ageing and adult health.Int J Geriatr Psychiatry. 2020; 35: 365-375.

22. Bourdieu P. The forms of capital. In Handbook of theory and research for the sociology of education. Edited by 
Richardson R. New York: Greenwood Press; 1986.

23. National Health Commission. China Migrant Population Development Report 2018. Beijing: China Population Press. 2019.

24. National Health Commission. National standards for Essential Public Health Services (Third Edition). 2017. http://www.National Health Commission.gov.cn/jws/s3578/201703/ d20c37e23e1f4c7db7 b8e25 f34473e1b.shtml. Accessed 15 Mar 2017.

25. National Health Commission. Program of pilot work on equalization of basic public services in health and household planning for migrant population. http://www.National Health Commission.gov.cn/ldrks/s3577/201312/ 39f344bd0a4f419ca66ef8b933eaa561.shtml. Accessed 19 Dec 2013.

26. The State Council. Interim Regulations on Residence Permits. http://www.gov.cn/zhengce/2015-12/14/ content_5023611. htm. Accessed 14 Dec 2015.

27. National Health Commission. Action Plan on Health Education and Promotion for the Migrant Population (2016-2020). http://www. National Health Commission.gov.cn/ldrks/s3577/201606/cf593583b37241a58068e0a a0b86d2de. shtml. Accessed 14 June 2016.

28. Zhang J, Lin S, Liang D, et al. Public health services utilization and its determinants among internal migrants in China: evidence from a nationally representative survey. International Journal of Environmental Research and Public Health. 2017; 14(9):1002.

29. Yin Q, Xu QL.Establishment of health records and its influencing factors among migrant populations. Chin J Public Health. 2018; 34(10): 1351-1355.

30. Zhang L, Zhou CC. Acquisition of tuberculosis knowledge and its relevant factors among intra-provincial and inter-provincial migrant populations in China. Chin J Public Health. 2019; 35(2):147-152.

31. Wei Y. Supply and utilization of health service among migrant population in China: a comparative study. Chin J Public Health. 2021; 37(2): 219-223.

32. Yan $\mathrm{Q}$, Tong L. Utilization of basic public health services and its influence factors among young migrants. Chin J Public Health. 2019; 35(6): 680-684.

33. Yang Z, Jiang CH.Impact of social capital, sex and education on the utilization of public health services: a cross sectional study based on the China migrant dynamic survey. BMC Public Health. 2021; 21:751.

34. Abraído-Lanza AF, Armbrister AN, Flórez KR, Aguirre AN. Toward a theory-driven model of acculturation in public health research. American Journal of Public Health. 2006; 96(8): 1342-1346.

35. Butler M, Warfa N, Khatib Y, Bhui K. Migration and common mental disorder: an improvement in mental health overtime. International Review of Psychiatry. 2015; 27(1): 51-63.

36. Yang LL, Zhang X. Dialect disparity, social assimilation and subjective well-being of floating population. Review of Investment Studies. 2019; 38(9): 125-135.

37. Xiao ZH, Xu SY, Liu JW. The assessment of social integration of urban migrant population: an Investigation based on 50 Cities of migration destination. Population Research. 2019; 43(5): 96-112.

38. Moore S, Carpiano RM. Measures of personal Social capital over time: a path analysis assessing longitudinal associations among cognitive, structural, and network elements of Social capital in women and men separately. Soc social capitali Med. 2020; doi: 10.1016/j.socscimed.2019.02.023.

39. Ren Y, Tao L. Localized social capital and social integration of migrants in urban China. Popul Res. 2012; 36(5): 47-57.

40. Palmer NA, Perkins DD. Social capital and community participation among migrant workers in China. Journal of community psychology. 2011; 39(1): 89 - 105.

41. Yip W, Subramanian SV, Mitchell AD, Lee DTS, Wang J, Kawachi I. Does social capital enhance health and well-being? Evidence from rural China. Soc Sci Med. 2007; 64(1): 35-49. https://doi.org/10.1016/j.socscimed. 
2006.08.027.

42. National Health Commission. China health statistical digest 2018. Beijing: Peking Union Medical College Press,2018.

43. Song LJ, Chang TY. Do resources of network members help in help seeking? social capital and health information search. Social Networks. 2012; 34 (2012): 658 - 669.

44. Wang YJ, Tong X. Analysis on the social support network of floating peasants.Sociological Studies. 2004; (2): 42-48. doi: 10.19934/j.cnki.shxyj.2004.02.004.

45. Lu Y, Ruan DC, Lai GN. Social capital and economic integration of migrants in urban China. Social Networks. 2013; 35 (2013): 357 - 369.

46. Viswanath K, Steele WR, Finnegan JR. Social Capital and Health: Civic Engagement, Community Size, and Recall of Health Messages. American Journal of Public Health. 2006; 96(8): 1456-1461.

47. Cohen S, Wills TA. Stress, social support, and the buffering hypothesis. Psychol Bull. 1985; 98: 310-357.

48. Fried $Y$, Tiegs R B.The main effect model versus buffering model of shop steward social support: a study of rank -and -file auto workers in the U.S.A. Journal of Organizational Behavior. 1993; 14: 5-12. 